\section{Alkoholabhängigkeit und psychiatrische Komorbidität - ein Überblick}

Jens Wittfoot, Martin Driessen

Psychiatrische Klinik, Bielefeld
Zusammenfassung: Zusätzliche psychische Störungen, insbesondere Angst- und affektive Störungen, finden sich bei Alkoholabhängigen in Behandlungseinrichtungen sehr viel häufiger als bei unbehandelten Abhängigen. Im Sinne des Komorbiditätskonzepts und auf der Grundlage operationalisierter Diagnostik zeigt bereits der zeitliche Zusammenhang ein komplexes Muster simultaner und sukzessiver Komorbidität. Eindeutige Belege für eine gemeinsame hereditäre bzw. genetische Grundlage von Abhängigkeit und komorbiden Störungen existieren nicht. So fokussiert sich das Interesse derzeit auf drei Fragen: 1. Stellen Alkoholabhängigkeit und komorbide psychische Störung wechselseitig Risikofaktoren für das Auftreten der jeweils anderen Erkrankung dar? 2. Beeinflussen komorbide Störungen und/oder das Ausmaß der aktuellen Psychopathologie den Verlauf der Abhängigkeit? 3. Sind komorbide psychische Störungen therapierelevant? Während Behandlungsprogramme für Alkoholabhängige mit komorbiden psychischen Störungen in der Praxis bereits existieren, fehlen bisher Untersuchungen zum Beleg der Wirksamkeit definierter pharmako-, psycho- und sozialtherapeutischer Interventionen.

Alcohol Dependence and Psychiatric Comorbidity - an Overview: Additional psychiatric disorders, i.e. mainly anxiety and affective disorders, were more frequently found in treated than in non treated alcohol-dependent persons. Basing on the concept of comorbidity operationalized diagnostic investigations revealed a complex pattern of successive and simultaneous comorbidity. A common hereditary and/or genetic origin of substance use disorders and comorbid psychiatric disorders cannot be stated. The current interest of researches and clinicians focusses on three issues: 1 . Are alcohol dependence and additional psychiatric disorders pathogenetic risk factors for each other? 2. Do comorbid psychiatric disorders and/or the degree of current psychopathology interact with the course of alcohol dependence? 3. Are comorbid disorders relevant for alcoholism treatment? While treatment programmes for comorbid alcoholics were introduced into the clinical practice, up to now, evidence is missing for the efficacy (and efficiency) of defined psychopharmacological, psychotherapeutical, and social interventions in the field.

Suchttherapie 2000; $1: 8-15$

(c) Georg Thieme Verlag Stuttgart · New York ISSN 1439-9903

\section{Einleitung und Begriffsbestimmung}

Die Bedeutung mehrerer Erkrankungen bei einer Person ist in der Psychiatrie erst im Verlauf der Hinwendung zu kriterienorientierter und operationalisierter Diagnostik in Forschung und Versorgung zunehmend erkannt und berücksichtigt worden. Insbesondere die deutschsprachige Psychiatrie war traditionell bemüht, im Sinne der von Schneider [1] und Jaspers [2] formulierten Schichtenregel auf die Person bezogene hierarchische Beziehungen der sichtbaren psychischen Symptome und Syndrome zu konstruieren. Der Interaktion zwischen Suchterkrankungen und nichtsubstanzbezogenen Störungen wurde dabei kaum Aufmerksamkeit geschenkt. Mit dem Komorbiditätskonzept, wie es zunächst im Bereich der somatischen Medizin entwickelt wurde [3], besteht die Möglichkeit, mehrere auf gleichem methodischen Niveau erfasste psychische Störungen bzw. Diagnosen auf ihre zeitliche Beziehung und auf ihre gegenseitige Einflussnahme hinsichtlich Prognose, Verlauf, Behandlungsmotivation etc. zu untersuchen [4]. Grundsätzlich kann hinsichtlich der zeitlichen Dimension simultane Komorbidität (das gleichzeitige Vorhandensein mehrerer Störungen) [5] von sukzessiver Komorbidität (dem zu unterschiedlichen Zeiten bei einer Person vorkommenden Auftreten verschiedener Störungen) unterschieden werden. Eine weitere Differenzierung stellt die Aufteilung in interne Komorbidität, bei der die vorliegenden Störungen derselben diagnostischen Klasse angehören, und $e x-$ terne Komorbidität, bei der Störungen aus unterschiedlichen Klassen vorliegen, dar. Besondere Fragestellungen ergeben sich aus der Einordnung von Persönlichkeitsstörungen in psychiatrischen Klassifikationssystemen. Ordnet man diese im Sinne des DSM IV [6] einer eigenen Achse (Achse 2) zu, ist es sinnvoll, Komorbidität unterschiedlicher Störungen der Achse 1 untereinander von Komorbidität einer Achse-1-Störung und einer Persönlichkeitsstörung zu differenzieren. Grundsätzlich ist Komorbidität nicht auf das Vorhandensein von zwei Störungen im Sinne einer „Doppeldiagnose“ begrenzt. Zunehmend wird vielmehr auf das Vorhandensein multipler psychiatrischer Komorbidität hingewiesen, wobei wiederum unterschiedliche Kombinationen aus interner/externer Komorbidität und unterschiedliche zeitliche Zusammenhänge vorliegen können.

\section{Epidemiologie}

Untersuchungen zur Komorbidität von Alkoholabhängigkeit bzw. Alkoholmissbrauch und anderen psychischen Störungen beziehen sich überwiegend auf stationär behandelte Patienten mit alkoholbezogenen Problemen, in geringerem Umfang auf 
die Allgemeinbevölkerung oder bestimmte Populationen. Gemessen wird dabei entweder die Aktualprävalenz (meist in Form der 6-Monats- oder 12-Monats-Prävalenz) oder die Lebenszeitprävalenz des Alkoholismus und der komorbiden psychischen Störung oder beides [4,7,8]. Die erhebliche Spannweite der gefundenen Prävalenzraten auch bei (fraglich) vergleichbaren Kollektiven ergibt sich aus der unterschiedlichen Berücksichtigung folgender Faktoren (Varianzquellen):

Es werden unterschiedliche diagnostische Instrumente verwendet.

- Alkoholabhängigkeit und -missbrauch werden häufig zusammengefasst (Alkoholismus).

- Alkoholkranke und Drogenabhängige werden nicht getrennt.

- Zusätzlich zum Alkoholismus bestehende substanzbezogene Störungen (interne Komorbidität z.B. mit drogenbezogenen Störungen) werden nicht mit erfasst.

- Die Untersuchungszeitpunkte stehen in unterschiedlicher Beziehung zum Abschluss der Detoxifikation (die Bedeutung dieser Varianzquelle ist allerdings nicht eindeutig).

Tab. 1 zeigt die Ergebnisse ausgewählter umfassender Prävalenzstudien zur Komorbidität im Bereich der Achse-1-Erkrankungen im Sinne des DSM-Systems (III, III-R, IV) aus dem psy- chiatrisch-stationären Bereich $[4,9,10,11,12,13]$. Deutlich wird v. a. der erhebliche Anteil von affektiven Störungen und Angststörungen bei zur Behandlung des Alkoholismus aufgenommenen Patienten. Bei internistisch oder chirurgisch behandelten Allgemeinkrankenhauspatienten fanden sich psychoorganische Störungen in einem erheblich höheren Ausmaß: Die Aktualprävalenz betrug in einer Untersuchung [14] $20 \%$ bei aktueller Diagnose einer Alkoholabhängigkeit und $16 \%$ bei den Patienten, die irgendwann während ihres Lebens die Kriterien für die Diagnose einer Alkoholabhängigkeit erfüllt hatten. Angststörungen und affektive Störungen unter den alkoholabhängigen Patienten fanden sich dagegen seltener als in vergleichbaren Untersuchungen an psychiatrisch behandelten Patienten (13,3\% bei aktueller Alkoholabhängigkeit). Prävalenzwerte zur psychiatrischen Komorbidität bei Alkoholabhängigen in der Allgemeinbevölkerung wurden u.a. in dem nordamerikanischen National Comorbidity Survey (NCS) bei 8098 Personen zwischen 15 und 54 Jahren zu Hause erhoben [15]. Dabei fand sich eine 12-Monats-Prävalenz für Alkoholabhängigkeit von $4,4 \%$ bei einer Lebenszeitprävalenz von $14,1 \%$. Die Lebenszeitprävalenz für irgendeine zusätzliche psychische Störung nach DSM-III-R betrug für Männer, die irgendwann im Leben eine Alkoholabhängigkeit entwickelt hatten, 78,3\%, für Frauen 86\%, wobei andere substanzbezo-

Tab. 1 Prävalenz komorbider Achse-I-Störungen nach DSM III bzw. ICD 10 bei psychiatrisch-stationär behandelten Patienten mit alkoholbezogenen Störungen. Angegeben ist die Aktualprävalenz in \% bzw. die Lebenszeitprävalenz in \%

\begin{tabular}{|c|c|c|c|c|c|c|}
\hline Autoren & $\begin{array}{l}\text { Hesselbrock et al. } \\
1985[9]\end{array}$ & $\begin{array}{l}\text { Ross et al. } 1988 \\
{[10]}\end{array}$ & $\begin{array}{l}\text { Herz et al. } 1990 \\
{[11]}\end{array}$ & $\begin{array}{l}\text { Tómasson u. } \\
\text { Vaglum } 1995 \text { [12] }\end{array}$ & $\begin{array}{l}\text { Driessen } 1999 \\
\text { [4] }\end{array}$ & $\begin{array}{l}\text { Schneider et al. } \\
\text { unveröff. [13] }\end{array}$ \\
\hline $\begin{array}{l}\text { Klassifikation } \\
\text { Instrumente }\end{array}$ & $\begin{array}{l}\text { DSM-III } \\
\text { DIS }\end{array}$ & $\begin{array}{l}\text { DSM-III } \\
\text { DIS }\end{array}$ & $\begin{array}{l}\text { DSM-III } \\
\text { DIS }\end{array}$ & $\begin{array}{l}\text { DSM-III } \\
\text { DIS }\end{array}$ & $\begin{array}{l}\text { ICD-10 } \\
\text { CIDI }\end{array}$ & $\begin{array}{l}\text { ICD-10 } \\
\text { Mini-DIPS }\end{array}$ \\
\hline $\mathrm{n}$ & 321 & 501 & 74 & 240 & 250 & 556 \\
\hline psychoorganische Störung & & & & & $3,2(3,2)$ & \\
\hline Schizophrenie & & & & & $2,0 \quad(2,4)$ & \\
\hline bipolare affektive Störung & & & & & $0,4 \quad(0,8)$ & \\
\hline depressive Episode & $23(38)$ & $20(24)$ & $(21)$ & (15) & $6,8 \quad(8,8)$ & 3,4 \\
\hline rezidiv. depressive Störung & & & & & $6,8 \quad(8,4)$ & 11,0 \\
\hline Dysthymia & & 11 (17) & (11) & (15) & $7,6(11,0)$ & 8,8 \\
\hline manische Störung & $2(4)$ & $2(2)$ & ( 3$)$ & ( 3$)$ & & \\
\hline Phobie & $18(27)$ & $20(34)$ & & & & \\
\hline Agoraphobie & & & & $(26 / 31)^{*}$ & $4,8 \quad(4,8)$ & 1,4 \\
\hline soziale Phobie & & & & $(24 / 33)^{*}$ & $7,6(8,8)$ & 13,7 \\
\hline spezifische Phobie & & & & $(21 / 29)^{*}$ & $14,0 \quad(17,0)$ & 18,5 \\
\hline Panikstörung & $6(10)$ & $9(10)$ & & $(4 / 13)^{*}$ & $1,2(2,0)$ & 5,2 \\
\hline $\begin{array}{l}\text { generalisierte Angststörung } \\
\text { Angststörung }\end{array}$ & & $26(52)$ & & $(31 / 43)^{*}$ & $2,8 \quad(4,0)$ & 12,9 \\
\hline sonstige Angststörung & & & & & $5,6(7,6)$ & \\
\hline Zwangsstörung & $5(12)$ & $6(10)$ & & $(5 / 7)^{*}$ & $1,6(1,6)$ & 2,2 \\
\hline dissoziative Störung & & & & & $2,0 \quad(4,0)$ & \\
\hline somatoforme Störung & & & & & $4,4 \quad(6,8)$ & 3,4 \\
\hline Anorexie & & & & & $0(0,4)$ & \\
\hline akute Belastungsreaktion & & & & & & 0,2 \\
\hline $\begin{array}{l}\text { posttraumat. Belastungs- } \\
\text { störung }\end{array}$ & & & & & & 6,1 \\
\hline
\end{tabular}

\footnotetext{
* Angaben getrennt für männl./weibl. Probanden

DIS=Diagnostic Interview Schedule

Mini-DIPS=Kurzfassung des diagnostischen Interviews bei psychischen Störungen
} 
gene Störungen eingeschlossen sind. Für Angststörungen (inklusive der posttraumatischen Belastungsstörung) ergab sich bezogen auf die Lebenszeitprävalenz eine Komorbiditätsrate von 35,8\% bei den Männern mit Alkoholabhängigkeit und $60,7 \%$ bei den Frauen, für affektive Störungen eine Rate von $28,1 \%$ bei den Männern und 53,5\% bei den Frauen. Auch aus der Oberbayern-Studie [16] lassen sich Hinweise auf zusätzliche psychiatrische Diagnosen (nach ICD-8) bei als alkoholkrank eingestuften Probanden finden. Danach betrug die Prävalenz komorbider Hauptdiagnosen 40,7\%. In der Verlaufsuntersuchung [17] fanden sich bei den Probanden mit Alkoholmissbrauch und -abhängigkeit (einschließlich der nicht behandlungsbedürftigen Probanden) in 9,5\% der Fälle zusätzlich neurotische Störungen, in 5,6\% der Fälle psychosomatische Erkrankungen, in 3,2\% der Fälle funktionelle Psychosen und in 3,2\% der Fälle Hinweise auf Hirnschädigungen.

Auch die Untersuchungen zur Prävalenz von Persönlichkeitsstörungen bei behandelten Patienten mit Alkoholmissbrauch bzw. Alkoholabhängigkeit zeigen gegenüber der Allgemeinbevölkerung erheblich erhöhte Prävalenzraten. In drei zwischen 1991 und 1993 publizierten Untersuchungen fanden sich Lebenszeitprävalenzen von 57 - 78\% für Persönlichkeitsstörungen nach DSM-III bzw. DSM-III-R $[18,19,20]$. In der auf alkoholabhängige Patienten ohne zusätzliche Substanzabhängigkeit beschränkten Lübecker Untersuchung [4] fanden sich bei $17,6 \%$ sichere und in weiteren $16,0 \%$ wahrscheinliche Persönlichkeitsstörungen. Tab. 2 zeigt die Befunde der vier Studien differenziert nach den Clustern A, B und C.

\section{Zusammenhang zwischen Alkoholismus und komorbiden psychischen Störungen}

Der Zusammenhang zwischen komorbiden Störungen kann grundsätzlich unter unterschiedlichen theoretischen Gesichtspunkten betrachtet werden [4]. Zunächst können zwei Störungen völlig unabhängig voneinander bei derselben Person auftreten (Zufallsmodell), was für jede beliebige Kombination von Störungen in einem bestimmten Ausmaß zu erwarten ist. Bei überzufällig häufigem gemeinsamen Auftreten kann nach gemeinsamen zugrunde liegenden Faktoren, vor allem im Sinne von hereditären Faktoren, gesucht werden. Beide Störungen wären dann Auswirkungen eines gemeinsamen zugrunde liegenden Prozesses (phänomenologisches Modell). Ferner können im Sinne eines ätiopathogenetischen Modells bzw. Risikofaktormodells Kausalzusammenhänge zwischen dem Auftreten der primär manifest werdenden Störung und dem Auftreten der sekundären Störung bestehen. Schließlich kann ein Zusammenhang auch ausschließlich die einseitige oder gegenseitige Verlaufsbeeinflussung zweier (oder mehrerer) komorbider Störungen im Sinne eines interaktionellen Modells betreffen.

In Bezug auf das Verhältnis zwischen Alkoholismus und komorbiden psychischen Störungen besteht seit dem Ende des 19.Jahrhunderts ein ständiger Wechsel zwischen der Annahme einer völligen Unabhängigkeit einerseits und der Hypothese einer gemeinsamen zugrunde liegenden Grundstörung andererseits [21]. Zunehmend werden jedoch in der Literatur differenziertere Modelle für das Verständnis unterschiedlicher Gruppen komorbider Alkoholkranker im Sinne der oben angegebenen Sichtweisen angenommen.

\section{Hereditäre Faktoren - phänomenologisches Modell}

Ein genereller genetischer Komorbiditätsfaktor als gemeinsame hereditäre oder genetische Grundlage für das Auftreten einer alkoholbezogenen Störung und irgendeiner komorbiden psychischen Störung scheint nach derzeitigem Forschungsstand unwahrscheinlich. Ebenso wenig gesichert ist die Annahme genetischer Faktoren für störungsspezifische Komorbiditätsmuster. Die Ergebnisse entsprechender Familien- und Zwillingsstudien sind widersprüchlich [22,23,24,25,26,27, 28,29]. Die Bedeutung eines angenommenen genetischen Faktors reduzierte sich vor allem immer dann, wenn die Ergebnisse auf familiäre bzw. individuumsspezifische Umgebungsfaktoren hin kontrolliert wurden.

\section{Risikofaktormodell}

Im Rahmen dieses Ansatzes kann, bezogen auf verschiedene komorbide Störungen, zumindest nach der zeitlichen Abfolge des erstmaligen Auftretens der jeweiligen Störung ein primärer Alkoholismus, der als Risikofaktor für eine sekundäre psychische Störung zu sehen ist, von einem sekundären Alkoholismus als Folge einer primären anderen psychiatrischen Erkrankung differenziert werden. Dieses Modell hat besondere Bedeutung für das Verständnis des Alkoholismus bei schizophrenen Patienten, der überwiegend als sekundäre Störung aufgefasst werden kann, während bei primärem Auftreten der Alkoholabhängigkeit diese bisher nicht als eigenständiger Risikofaktor für das sekundäre Auftreten einer schizophrenen Erkrankung identifiziert werden konnte [10,30].

Alkoholabhängigkeit als unmittelbarer Risikofaktor ist hingegen für das Auftreten einer komorbiden depressiven Störung zu diskutieren. So kann sich z.B. eine sekundäre Dysthymia als Folge gelernter Hilflosigkeit angesichts der Ohnmacht gegenüber der Unkontrollierbarkeit der Abhängigkeit entwickeln, während dysphorische Syndrome eher als Intoxikationsfolge bzw. in der frühen Entzugsphase auftreten und als psychoorganisches Syndrom verstanden werden können [31,32,33]. Schuckit et al. [34] fassen alle sekundären Depressionen grundsätzlich als alkohol- bzw. alkoholismusinduziert auf. Über ein andererseits möglicherweise erhöhtes Alkoholismusrisiko bei primären depressiven Störungen ist wenig bekannt. Gezeigt werden konnte jedoch, dass Frauen mit Alkoholismus und depressiver Störung häufiger primär unter der depressiven Störung litten, bei den komorbid erkrankten Männern dagegen der überwiegende Teil den Alkoholismus primär entwickelte [35].

Angststörungen, insbesondere Phobien, scheinen zum überwiegenden Teil primär aufzutreten $[4,9,10,36]$. Verständlich wird dies durch die anxiolytische Wirkung des Alkohols bzw. die subjektive Erwartung einer solchen Wirkung [37]. Andererseits scheint der Langzeiteffekt des Alkohols eher in einer Steigerung des Angstniveaus zu liegen [38], was mit metabolischen Folgen der chronischen Alkoholintoxikation und mit wiederholter Erfahrung angstbesetzter Entzugssyndrome in Verbindung gebracht werden kann [39]. Die komplexe Interaktion zwischen Alkoholabhängigkeit, Alkoholentzugssituation und Angststörung kommt in einer Untersuchung von Roberts et al. [40] zum Ausdruck, bei der der Anteil der alkoholabhängigen Patienten, die aktuell (Punktprävalenz) die formalen Kriterien für eine Angststörung nach DSM-IV erfüll- 
ten, von 22\% während der stationären Entgiftung auf $10 \%$ nach drei bis vier Wochen Abstinenz abfiel. Andererseits berichten alkoholabhängige Patienten mit komorbiden Angststörungen drei bis sieben Wochen nach Abstinenzbeginn immer noch deutlich mehr Angstsymptome als Alkoholabhängige ohne komorbide Störung [41, eigene unveröffentlichte Ergebnisse].

Für eine erhebliche Wechselwirkung zwischen Angststörungen und Alkoholabhängigkeit im Sinne eines Risikofaktormodells sprechen auch die Ergebnisse einer umfangreichen prospektiven Studie von Kushner et al. [42]. Dabei wurden Studenten im Jahr ihres Studienbeginns sowie nach vier und sieben Jahren auf die 12-Monats-Prävalenz von Angststörungen und Alkoholabhängigkeit bzw. Alkoholmissbrauch nach DSM-IV-Kriterien hin untersucht. Das Risiko, im Untersuchungszeitraum eine Alkoholabhängigkeit zu entwickeln, war bei den Untersuchten mit Vorhandensein einer Angststörung im Jahr 1 oder 4 etwa viermal so hoch wie bei den Probanden ohne Angststörung. Umgekehrt führte das Vorhandensein einer Alkoholabhängigkeit im Jahr 1 oder 4 zum 3,5- bis 5 fachen Risiko für die Entwicklung einer Angststörung.

Bei den Achse-II-Störungen ist insbesondere die antisoziale Persönlichkeitsstörung (ASP) in ihrer Beziehung zum Alkoholismus häufig untersucht worden. Viele Autoren sehen bei Alkoholabhängigen mit ASP eine rasche und früh einsetzende Abhängigkeitsentwicklung, was die ASP als einen Risikofaktor für die Entwicklung einer bei dieser Patientengruppe häufig anzutreffende atypische bzw. Typ-2-Alkoholabhängigkeit ausweisen könnte $[11,43,44,45,46,47,48,49,50,51,52]$. Entsprechende Hinweise für das frühere Auftreten der Alkoholabhängigkeit finden sich auch für die Borderline-Persönlichkeitsstörung, allerdings nur für Frauen [53]. Andere spezifische Persönlichkeitsstörungen sind in dieser Hinsicht nicht ausreichend untersucht worden.

Besondere Bedeutung hat die Frage nach der gegenseitigen Beziehung von Alkoholismus, Achse-I-Störungen und AchseII-Persönlichkeitsstörungen. In der Lübecker Komorbiditätsstudie konnte gezeigt werden, dass die Prävalenz der affektiven Störungen und der Angststörungen nur noch halb so hoch war, wenn die Patienten mit zusätzlichen Persönlichkeitsstörungen ausgeschlossen würden. Persönlichkeitsstörungen könnten daher als grundsätzlicher Risikofaktor für das Auftreten einer komorbiden Achse-I-Störung bei Alkoholismus angesehen werden [4].

\section{Gegenseitige Verlaufsbeeinflussung - Interaktionales Modell}

Schizophrene Störungen verlaufen ungünstiger, führen zu häufigeren Hospitalisierungen und damit mehr Kosten und gehen mit erhöhter Suizidalität einher, wenn eine zusätzliche alkoholbezogene Störung (und häufig auch zusätzlicher pathologischer Drogengebrauch) vorhanden ist. Dahingegen liegen über den Verlauf einer Alkoholabhängigkeit bei Vorliegen einer zusätzlichen schizophrenen Störung weniger sichere Erkenntnisse vor $[54,55,56,57,58,59,60]$.

Depressive Störungen scheinen bei gleichzeitigem Alkoholismus hinsichtlich einer geringeren Remissionsneigung ungünstiger zu verlaufen, zumindest verbessert eine Remission der Alkoholkrankheit die Verlaufsprognose einer gleichzeitig bestehenden Depression [61]. Ein klinisch erheblich relevantes Problem bei Alkoholismus und komorbiden psychischen Störungen, insbesondere bei depressiven Störungen, ist die erhöhte Suizidalität. Murphy et al. [62] fanden unter 50 suizidierten Alkoholikern bei 68\% die Kriterien einer Major Depression nach DSM-III-R erfüllt. Für die Suizidversuchsrate bei Alkoholikern fanden Biro et al. [63] einen Anstieg von 9,5 auf $17,3 \%$, wenn eine zusätzliche depressive Störung vorlag, Roy et al. [35] konnten einen Anstieg von 12 auf 32\% darstellen. Parallel dazu lässt sich auch zeigen, dass das Vorliegen einer Alkoholabhängigkeit bei Patienten mit einer depressiven Störung zu einem erheblich größeren Ausmaß von Suizidalität und negativem Selbsterleben führt [64]. Sowohl für die Suizidrate als auch für die Suizidversuchsrate scheint außerdem das Vorliegen einer antisozialen Persönlichkeitsstörung einen zusätzlichen Risikofaktor darzustellen [65]. Außer depressiven Störungen scheinen auch Angststörungen das Risiko suizidaler Krisen erheblich zu erhöhen und insbesondere eine multiple Komorbidität (Angststörung und depressive Störung bzw. Achse-I-Störung und Achse-II-Störung im Sinne des DSM-Systems) mit dem größten Suizidrisiko einherzugehen [66]. Pirkola et al. [67] konnten zeigen, dass sich Suizidanten der Altersgruppe 13 bis 22 Jahre, bei denen eine alkoholbezogene Störung retrospektiv angenommen werden konnte, von den Suizidanten ohne alkoholbezogene Störung durch zusätzliche psychiatrische Komorbidität, antisoziale Verhaltensmuster, zerrüttete Familienverhältnisse, belastende Lebensereignisse und ernsthafte psychosoziale Defizite unterschieden.

Aus den Befunden lässt sich ein Modell ableiten, in dem primäre oder sekundäre komorbide depressive Störungen die Hauptrisikofaktoren für Suizidalität bei Alkoholismus sind. Dem Alkoholismus (und dessen psychosozialen Folgen) sowie (durch den Alkoholismus begünstigten) belastenden Lebensereignissen und interpersonalen Konflikten käme damit eher eine modifizierende Funktion z.B. im Sinne der forcierten Umsetzung suizidaler Impulse zu [4].

Untersuchungen über den Verlauf der Alkoholabhängigkeit selbst bei gleichzeitigem Vorliegen einer depressiven Störung sind uneinheitlich. Während mehrere Studien keine relevanten Unterschiede im Vergleich zu nicht depressiven Patienten fanden $[4,9,68]$, ergaben sich in anderen Untersuchungen Hinweise auf einen ungünstigeren Verlauf der Suchterkrankung bzw. auf schwerere suchtbezogene Symptome bei männlichen Alkoholkranken mit einer zusätzlichen depressiven Störung $[49,69]$. Andererseits berichten Kranzler et al. [70] sogar über einen günstigeren Verlauf des Alkoholismus bei Vorliegen einer Major Depression. Über den Krankheitsverlauf der einzelnen Störungen beim komorbiden Auftreten von Alkoholabhängigkeit und Angststörungen gibt es bisher nur wenige Ergebnisse. Thomas et al. [71] konnten zeigen, dass alkoholabhängige Patienten mit sozialer Phobie sich von Alkoholabhängigen ohne soziale Phobie unter anderem durch ihre Trinkmuster und durch erhöhte Depressivität unterscheiden, jedoch nicht durch stärkeren Alkoholkonsum. Tomasson und Vaglum [72] berichten über eine deutlich höhere Frequenz stationärer Wiederaufnahmen von Alkoholikern bei zusätzlicher Agoraphobie oder Panikstörung. Bei antisozialen Persönlichkeitsstörungen wird ein ungünstigerer Verlauf der Abhängigkeitserkrankung beschrieben $[11,43,44,45,46,47$, $48,49,50,51,70,73]$, ebenso bei Borderline-Persönlichkeits- 
Tab. 2 Lebenszeitprävalenz spezifischer Persönlichkeitsstörungen bei Alkohomissbrauch und -abhängigkeit in \%

\begin{tabular}{|c|c|c|c|c|}
\hline & Nace et al. 1991 [18] & Smyth 1993 [19] ${ }^{a}$ & Dejong et al. 1993 [20] & Driessen 1999 [4] \\
\hline $\begin{array}{l}\text { Klassifikation } \\
\text { Instrumente }\end{array}$ & $\begin{array}{l}\text { DSM-III-R } \\
\text { SCID-II }\end{array}$ & $\begin{array}{l}\text { DSM-III-R } \\
\text { SCID-II }\end{array}$ & $\begin{array}{l}\text { DSM- III } \\
\text { SIDP }\end{array}$ & $\begin{array}{l}\text { DSM-III-R } \\
\text { IPDE }\end{array}$ \\
\hline$n$ & 100 & 50 & 178 & 250 \\
\hline $\begin{array}{l}\text { Cluster A } \\
\text { paranoid } \\
\text { schizoid } \\
\text { Schizotyp }\end{array}$ & $\begin{array}{l}7 \\
0 \\
0\end{array}$ & $\begin{aligned} 34 & (6) \\
2 & (0) \\
6 & (2)\end{aligned}$ & $\begin{aligned} 14 & (16) \\
4 & (14) \\
17 & (7)\end{aligned}$ & $\begin{array}{l}1,2 \\
4,3 \\
0,8\end{array}$ \\
\hline $\begin{array}{l}\text { Cluster B } \\
\text { antisozial } \\
\text { Borderline } \\
\text { histrionisch } \\
\text { narzisstisch }\end{array}$ & $\begin{array}{r}3 \\
17 \\
6 \\
4\end{array}$ & $\begin{array}{rr}14 & (8) \\
32 & (20) \\
34 & (23) \\
7 & (25)\end{array}$ & $\begin{array}{rr}5 & (0) \\
17 & (7) \\
34 & (23) \\
7 & (25)\end{array}$ & $\begin{array}{l}4,4 \\
3,2 \\
0,8 \\
0,4\end{array}$ \\
\hline $\begin{array}{l}\text { Cluster C } \\
\text { selbstunsicher } \\
\text { abhängig } \\
\text { zwanghaft } \\
\text { passiv-aggressiv }\end{array}$ & $\begin{array}{l}2 \\
4 \\
2 \\
5\end{array}$ & $\begin{array}{ll}32 & (10) \\
14 & (6) \\
12 & (4) \\
20 & (2)\end{array}$ & $\begin{array}{ll}19 & (12) \\
29 & (23) \\
19 & (18) \\
14 & (8)\end{array}$ & $\begin{array}{l}5,2 \\
2,4 \\
0,8 \\
0\end{array}$ \\
\hline $\begin{array}{l}\text { selbstschädigend } \\
\text { nicht spezifisch }\end{array}$ & $\begin{array}{l}5 \\
2\end{array}$ & $\overline{2}(2)$ & $\begin{array}{l}- \\
-\end{array}$ & 16,8 \\
\hline
\end{tabular}

a Hauptdiagnose in ()

${ }^{b}$ Anteil ohne weitere Persönlichkeitsstörung in ()

SCID-II=Structured Clinical Interview for DSM

SIDP=???

IPDE = International Personality Disorder Examination

störungen [53]. Zu anderen spezifischen Persönlichkeitsstörungen liegen kaum Verlaufsuntersuchungen vor. Für Persönlichkeitsstörungen insgesamt konnte jedoch in der Lübecker Komorbiditätsstudie ein deutlich schlechterer Verlauf der Abhängigkeitserkrankung sowohl im Vergleich zur Gruppe der nicht-komorbiden Patienten als auch im Vergleich zur Gruppe der Patienten mit ausschließlicher Achse-I-Komorbidität gezeigt werden. Dies galt jedoch nicht für die Untergruppe der Cluster-C-Persönlichkeitsstörungen. Der Verlauf bei den Patienten mit komorbider Achse-I-Störung ohne zusätzliche Persönlichkeitsstörung ließ sich dagegen praktisch nicht vom Verlauf bei den Patienten ohne komorbide Störung unterscheiden. Damit ergibt sich ein deutlicher Hinweis für die erhebliche prognostische Bedeutung von Persönlichkeitsstörungen, insbesondere der Cluster A und B [4].

\section{Therapie von Alkoholismus und Komorbidität}

Patienten mit substanzbezogenen Erkrankungen und komorbiden psychischen Störungen nehmen häufiger allgemeinmedizinische, psychiatrische und suchttherapeutische Hilfen in Anspruch und verursachen dadurch mehr Kosten als abhängige Patienten ohne zusätzliche Störungen und auch als Patienten mit psychischen Störungen ohne Abhängigkeit [74,75].

Angesichts dieser Situation sind die Entwicklung und Evaluation spezifischer Therapieprogramme dringend notwendig. Tatsächlich hat die Wahrnehmung der Problemlage durch die Kliniker insbesondere in Fachkrankenhäusern für Abhängigkeitserkrankungen in Deutschland und in angloamerikanischen Einrichtungen zur Implementierung von therapeutischen Angeboten z.B. für komorbide Angst- oder depressive Patienten geführt. Diesen pragmatischen Lösungen steht allerdings kaum empirisch abgesichertes Wissen gegenüber. Moggi et al. [76,77] definierten auf der Grundlage früherer Befunde solche Behandlungsprogramme als förderlich für Doppeldiagnosepatienten („dual diagnosis treatment climate“), die durch Anteilnahme, eine supportive Atmosphäre, praktische Orientierung, ein hohes Maß an Ordnung und Organisation und Klarheit des Programms gekennzeichnet waren. Sie fanden allerdings nur einen mäßigen positiven Zusammenhang zwischen diesen Therapieprogrammmerkmalen einerseits und substanzspezifischen Copingmustern der Patienten, Reduzierung der psychiatrischen Symptombelastung und Beschäftigungsrate 12 Monate nach Entlassung andererseits. Diese und andere Untersuchungen geben zwar allgemeine Hinweise auf eine mögliche Wirksamkeit von Komorbiditäts- oder Doppeldiagnoseprogrammen, sie sind aber in mehrfacher Hinsicht nicht ausreichend, um Aussagen über bestimmte Therapieformen für komorbide Alkoholabhängige im Vergleich zu konventionellen Alkoholismustherapien zu treffen: So wurden z.B. Alkohol- und Drogenabhängige bzw. polyvalent Abhängige einerseits und schizophrene und „neurotische“ Patientengruppen andererseits gemeinsam untersucht.

In psychopharmakologischen Studien bei Alkoholabhängigen wurde wiederholt die Wirksamkeit von trizyklischen Antidepressiva, Serotonin-Wiederaufnahmehemmern und Buspiron zur Frage der Reduktion von Depressivität und Angst in den ersten Wochen nach Entzugsbehandlung untersucht. Die Ergebnisse sind widersprüchlich, so dass von einer eindeutigen Überlegenheit gegenüber Plazebo generell nicht ausgegangen werden kann [78,79,80,81]. Ebenso wenig lässt sich die Frage nach der Wirksamkeit entsprechender pharmakologischer Interventionen auf günstigere alkoholbezogene Verhaltensweisen oder gar Abstinenz beantworten [79,82,83,84, 85]. Unglücklicherweise wurden bei diesen Studien komorbide Störungen nicht explizit erfasst, so dass offen bleibt, ob 
Patienten mit einer aktuellen bzw. früheren Diagnose einer depressiven oder Angststörung oder Patienten mit ausgeprägter Depressivität bzw. Angst auch nach Abschluss der Detoxifikation nicht doch von psychopharmakologischen und/oder gezielten psychotherapeutischen Interventionen profitieren können.

\section{Perspektiven}

Während die epidemiologischen Daten zur Komorbidität des Alkoholismus vorläufig ausreichend empirisch abgesichert sind, fehlen zur Zeit trotz der z.T. verwirrenden Vielfalt von Einzelbefunden spezifische Kenntnisse zum Zusammenhang von Alkoholismus und Komorbidität. Allerdings sind hier in nächster Zeit insbesondere aus der Stressforschung neue Ergebnisse zu erwarten, die den Zusammenhang von Angst und alkoholbezogenem Verhalten - möglicherweise diagnosenunspezifisch - besser verständlich machen. Die größte Unklarheit besteht allerdings nach wie vor zur Frage der Art, Organisation, Effektivität und Effizienz von komorbiditätsspezifischen Therapiemodellen.

\section{Literatur}

1 Schneider K. Psychischer Befund und psychiatrische Diagnose. Leipzig: Thieme, 1939

2 Jaspers K. Allgemeine Psychopathologie. Berlin, Heidelberg: Springer, 1948

${ }^{3}$ Feinstein AR. The pretherapeutic classification of comorbidity in chronic disease. Am J Psychiatry 1970; 142: 1259-1264

${ }^{4}$ Driessen M. Psychiatrische Komorbidität bei Alkoholismus und Verlauf der Abhängigkeit. Darmstadt: Steinkopff, 1999

${ }^{5}$ Kosten T, Kleber H. Differential diagnosis of psychiatric comorbidity in substance abusers. J Subst Abuse Treat 1988; 5: 201-206

${ }^{6}$ American Psychiatric Association. Diagnostic and Statistical Manual of Mental Disorders. Darmstadt: Steinkopff, 1999

${ }^{7}$ RachBeisel J, Scott J, Dixon L. Co-occurring severe mental illness and substance use disorders: a review of recent research. Psychiatr Serv 1999; 5: 1427-1434

${ }^{8}$ Brady KT. Comorbidity of substance use and axis I psychiatric disorders. Medscape Mental Health, 1998: 3

${ }^{9}$ Hesselbrock MN, Meyer RE, Keener JJ. Psychopathology in hospitalized alcoholics. Arch Gen Psychiatry 1985; 42: 1050-1055

${ }^{10}$ Ross HE, Glaser FB, Germanson T. The prevalence of psychiatric disorders in patients with alcohol and other drug problems. Arch Gen Psychiatry 1988; 45: 1023-1031

${ }^{11}$ Herz L, Volicer R, D'Angelo N, Gadish D. Additional psychiatric illness by Diagnostic Interview Schedule in male alcoholics. Compr Psychiatry 1990; 3: 72-79

12 Tomasson K, Vaglum P. A nationwide representative sample of treatment-seeking alcoholics: a study of psychiatric comorbidity. Acta Psychiatr Scand 1995; 92: 378-385

${ }^{13}$ Schneider $U$ et al. Comorbid anxiety and affective disorders on alcohol dependent patients seeking treatment: the forst multicenter study in Germany. Unveröffentlicht,

${ }^{14}$ Arolt V, Driessen M. Alcoholism and psychiatric comorbidity in general hospital inpatients. Gen Hosp Psychiatry 1996; 18: 271 277

15 Kessler RC, Crum RM, Warner LA, Nelson CB, Schulenberg J, Anthony JC. Lifetime co-occurrance of DSM-III-R alcohol abuse and dependence with other psychiatric disorders in the National Comorbidity Survey. Arch Gen Psychiatry 1997; 54: 313-321

${ }^{16}$ Dilling H, Weyerer S. Psychische Erkrankungen in der Bevölkerung bei Erwachsenen und Jugendlichen. In: Dilling $\mathrm{H}$, Weyerer
S, Castell R (Hrsg). Psychische Erkrankungen in der Bevölkerung, Stuttgart: Enke, 1984: 1-121

17 Fichter MM. Prävalenz von Alkoholabusus und -abhängigkeit in der Bevölkerung. In: Fichter MM (Hrsg). Verlauf psychischer Erkrankungen in der Bevölkerung, Berlin, Heidelberg, New York: Springer, 1990: 72-83

18 Nace EP, Davis CW, Gaspari JP. Axis II comorbidity in substance abusers. Am J Psychiatry 1991; 148: 118-120

${ }^{19}$ Smyth NJ. Differences in situational heavy drinking among alcoholics with and without comorbid personality disorders. VI Congress on the Treatment of Addictive Behavior 1993. Santa Fé, USA: Abstract, 1993

${ }^{20}$ DeJong AJ, van den Brink W, Hartefeld FM, van der Wielen EGM. Personality disorders in alcoholics and drug addicts. Compr Psychiatry 1993 ; 34: 87-94

${ }^{21}$ Parker JB, Meiller RM, Andrews GW. Major psychiatric disorders masquerading as alcoholism. South Med J 1960; 53: 560-565

22 Merikangas K, Risch NJ, Weissman MM. Comorbidity and cotransmission of alcoholism, anxiety and depression. Psychological Medicine 1994; 24: 69-80

${ }^{23}$ Kendler K, Heath H, Neale M, Kessler R, Eaves L. Alcoholism and major depression in women. A twin study of the causes of comorbidity. Arch Gen Psychiatry 1993; 5: 690-698

${ }^{24}$ Pickens RW, Svikis DS, McGue M, LaBuda MC. Common genetic mechanisms in alcohol, drug, and mental disorder comorbidity. Drug Alcohol Depend 1995 39: 129-138

${ }^{25}$ Maier W, Merikangas K. Co-occurrence and contransmission of affective disorders and alcoholism in families. BJP 1996; 3: 93100

${ }^{26}$ Kendler KS, Davis CG, Kessler RC. The familial aggregation of common psychiatric and substance use disorders in the National Comorbidity Survey: a family history study. BJP 1997; 17: 541548

27 Dinwiddie SH, Reich T. Genetic and family studies in psychiatric illness and alcohol and drug dependence. J Addict Disord 1993 12: $17-27$

${ }^{28}$ Kaye WH, Lilenfeld LR, Plotnicov K, Merikangas KR, Nagy L, Strober M, Bulik CM, Moss H, Greeno CG. Bulimia nervosa and substance dependence: association and family transmission. Alc Clin Exp Res 1996; 2: 878-881

${ }^{29}$ Kendler KS, Walters EE, Neale MC, Kessler RC, Heath AC, Eaves LJ. The structure of the genetic and environmental risk factors for six major psychiatric disorders in women. Phobia, generalized anxiety disorder, panic disorder, bulimia, major depression, and alcoholism. Arch Gen Psychiatry 1995; 52: 374-383

${ }^{30}$ Hambrecht M, Haefner H. Führen Alkohol- oder Drogenmissbrauch zu Schizophrenie. Nervenarzt 1996; 67: 36-45

${ }^{31}$ Behar D, Winokur G, Berg CJ. Depression in the abstinent alcoholic. Am J Psychiatry 1984; 141: 1105-1107

32 Schuckit M. Alcoholic patients with secondary depression. Am J Psychiatry 1983; 14: 711-714

33 Schuckit M. Genetic and clinical implications of alcoholism and affective disorder. Am J Psychiatry 1986; 143: 140-147

${ }^{34}$ Schuckit MA, Tipp JE, Bergman M, Reich W, Hesselbrock VM, Smith TL. Comparison of induced and independent major depressive disorders in 2,945 alcoholics. Am J Psychiatry 1997; 154: 948-957

${ }^{35}$ Roy A, DeJong J, Lamparski D, George T, Linnoila M. Depression among alcoholics. Arch Gen Psychiatry 1991; 48: 428-432

${ }^{36}$ Johannessen D, Cowley D, Walker R, Jensen C, Parker L. Prevalence, onset, and clinical recognition of panic states in hospitalized male alcoholics. Am J Psychiatry 1989; 146: 1201-1203

37 Kuefner H. Angst und Alkohol. In: Faust V (Hrsg). Angst-FurchtPanik, Stuttgart: Hippokrates, 1986: 141-147

38 Wiseman EJ. Alcohol dependence may be the missing link between posttraumatic stress disorder and panic. Arch Gen Psychiatry $1994 ; 51: 429-430$ 
39 Baving L, Olbrich H. Anxiety in alcohol dependent patients. Fortschr Neurol Psychiat 1996 64: 83-89

${ }^{40}$ Roberts MC, Emsley RA, Pienaar WP, Stein DJ. Anxiety disorders among abstinent alcohol-dependent patients. Psychiatr Serv 1999; 5: 1359-1361

41 Thevos AK, Johnston AL, Latham PK, Randall CL, Adinoff B, Malcolm R. Symptoms of anxiety in inpatient alcoholics with and without DSM-III-R anxiety diagnoses. Alc Clin Exp Res 1991; 15: 102-105

${ }^{42}$ Kushner MG, Sher KJ, Erickson DJ. Prospective analysis of the relation between DSM-III anxiety disorders and alcohol use disorders. Am J Psychiatry 1999; 156: 723-732

43 Schuckit MA. Alcoholism and sociopathy. Diagnostic confusion. J Stud Alcohol 1973; 34: 157-164

44 Tarter RE, McBride H, Buonpane N, Schneider DU. Differentiation of alcoholics. childhood history of minimal brain dysfunction, family history, and drinking pattern. Arch Gen Psychiatry 1979; 34: 761-768

45 Virkkunen M. Alcoholism and antisocial personality. Acta Psychiatr Scand 1979 59: 493-501

${ }^{46}$ Rada RT. Alcoholism and sociopathy: Diagnostic and Treatment Implications. In: Kaufman E (Hrsg). Encyclopedic Handbook of Alcoholism, New York: Gardner, 1982: 647-654

${ }^{47}$ Lewis CE, Rice J, Helzer JE. Diagnostic interactions between alcoholism and antisocial personality. J Nerv Ment Dis 1983; 171 105-113

48 Hesslbrock MN. Childhood Behavior Problems and Adult Antisocial Personality Disorder in Alcoholism. In: Meyer RE (Hrsg). Psychopathology and Addictive Disorders, New York, London: Guilford, 1986: 78-93

${ }^{49}$ Rounsaville BJ, Dolinsky CS, Babor TF, Meyer RE. Psychopathology as a predictor of treament outcome in alcoholics. Arch Gen Psychiatry $1987 ; 44:$ 505-513

${ }^{50}$ Alterman AI, Gerstley LJ, Strohmetz DB, McKay JR. Psychiatric Heterogeneity in antisocial alcoholics: relation to familial alcoholism. Compr Psychiatry 1991; 32: 423-430

51 Powell B, Penick E, Nickel E, Liskow BI, Riesenmy KE, Campion SL, Brown EF. Outcomes of co-morbid alcoholic men: a 1-year follow-up. Alc Clin Exp Res 1992; 16: 131-138

52 McGue M, Slutske W, Taylor J, Iacono WG. Personality and substance use disorders: I. Effects of gender and alcoholism subtype. Alc Clin Exp Res 1997 21: 513-520

${ }^{53}$ von der Stein B, Podoll K. Borderline-Persönlichkeitsstörung bei Patienten mit chronischem Alkoholismus. Sucht 1992; 2: 99106

54 Bartels S, Drake RE, McHugo GJ. Alcohol abuse, depression, and suicidal behavior in schizophrenia. Am J Psychiatry 1992; 149: 394-395

55 Cuffel BJ, Chase P. Remission and relapse of substance use disorders in schizophrenia. J Nerv Ment Dis 1994; 182: 342-348

56 Drake RE, Wallach MA. Moderate drinking among people with severe mental illness. Hospital and Community Psychiatry 1993; 4: $1041-1046$

57 Soyka M. Sucht und Schizophrenie. Nosologische, klinische und therapeutische Fragestellungen. 1. Alkoholismus und Schizophrenie. Fortschr Neurol Psychiat 1994; 62: 71-87

${ }^{58}$ Chouljian TL, Shumway M, Balancio E, Dwyer EV, Surber R, Jacobs M. Substance use among schizophrenic outpatients: prevalence, course, and relation to functional status. Ann Clin Psychiat 1995; $7: 19-24$

${ }^{59}$ Kozaric-Kovacic D, Folnegovic-Smalc V, Folnegovic Z, Maruic A. Influence of alcoholism on the prognosis of schizophrenic patients. J Stud Alcohol 1995; 56: 622-627

60 Uchtenhagen A. Schizophrenia and substance dependence. Schw Arch Neuro Psychiat 1995; 146: 215-223
61 Hasin DS, Tsai WY, Endicott J, Mueller TI, Coryell W, Keller M. Five-year course of major depression: effects of comorbid alcoholism. J Affect Disord 1996; 41: 63-70

62 Murphy GE, Armstrong JW, Hermele SL, Fischer JR, Clendenin WW. Suicide and alcoholism: interpersonal loss confirmed as a predictor. Arch Gen Psychiatry 1979; 36: 65-69

63 Biro M, Selakovic-Bursic S, Kapamadzija B. The role of depressive disorder in the suicidal behavior of alcoholics. Crisis 1991; 12: 64-68

${ }^{64}$ Cornelius JR, Salloum IM, Mezzich J, Cornelius MD, Fabrega H Jr, Ehler JG, Ulrich RF, Thase ME, Mann JJ. Disproportionate suicidality in patients with comorbid major depression and alcoholism. Am J Psychiatry 1995; 152: 358-364

65 Whitters A, Cadoret R, Troughton E, Widmer R. Suicide attempts in antisocial alcoholics. The Journal of Nervous and Mental Disease. J Nerv Ment Dis 1987; 175: 626-626

${ }^{66}$ Driessen M, Veltrup C, Weber J, John U, Wetterling T, Dilling H. Psychiatric co-morbidity, suicidal behaviour and suicidal ideation in alcoholics seeking treatment. Addiction 1998; 93: 889894

67 Pirkola SP, Marttunen MJ, Henriksson MM, Isometsae ET, Heikkinen ME, Loennqvist ME. Alcohol-related problems among adolescent suicides in Finland. Alcohol \& Alcoholism 1999; 34: 320329

${ }^{68}$ Driessen M, Arolt V, John U, Veltrup C, Dilling H. Psychiatric comorbidity in hospitalized alcoholics after detoxification treatment. Europ Addict Res 1996; 2: 17-23

69 Greenfield SF, Weiss RD, Muenz LR, Vagge LM, Kelly JF, Bello LR, Michael J. The effect of depression on return to drinking: a prospective study. Arch Gen Psychiatry 1998; 55: 259-265

70 Kranzler HR, Del Boca FK, Rounsaville BJ. Comorbid psychiatric diagnosis predicts three-year outcomes in alcoholics: a posttreatment natural history study. J Stud Alcohol 1996; 57: 619626

71 Thomas SE, Thevos AK, Randall CL. Alcoholics with and without social phobia: a comparison of substance use and psychiatric variables. J Stud Alcohol 1999; 6: 472-479

72 Tomasson K, Vaglum P. Role of psychiatric comorbidity in the prediction of readmission for detoxification. Compr Psychiatry 1998; 39: 129-136

${ }^{73}$ Holdcraft LC, Iacono WG, McGue MK. Antisocial Personality Disorder and depression in relation to alcoholism: a communitybased sample. J Stud Alcohol 1998; 59: 222-226

${ }^{74}$ Hoff RA, Rosenheck RA. The cost of treating substance abuse patients with and without comorbid psychiatric disorders. Psychiatr Serv 1999; 5: 1309-1315

75 Maynard C, Cox GB. Psychiatric hospitalization of persons with dual diagnoses: estimates from two national surveys. Psychiatr Serv 1998; 49: 1615-1617

${ }^{76}$ Moggi F, Ouimette PC, Finney JW, Moos RH. Effectiveness of treatment for substance abuse and dependence for dual diagnosis patients: a model of treatment factors associated with oneyear outcomes. J Stud Alcohol 1999; 6: 856-866

77 Moggi F, Ouimette PC, Moos RH, Finney JW. Dual diagnosis patients in substance abuse treatment: relationship of general coping and substance-spezific coping to 1-year outcomes. Addiction 1999; 94: 1805-1816

78 George DT, Nutt DJ, Dwyer BA, Linnoila M. Alcoholism and panic disorder: is the comorbidity more than coincidence?. Acta Psychiatr Scand 1990; 81: 97-107

${ }^{79}$ Kranzler HR, Burleson JA, Brown J, Babor TF. Fluoxetine treatment seems to reduce the beneficial effects of cognitive-behavioral therapy in type B alcoholics. Alc Clin Exp Res 1996; 2: 15341541

${ }^{80}$ Lejoyeux M. Use of serotonin (5-hydroxytryptamine) reuptake inhibitors in the treatment of alcoholism. Alcohol \& Alcoholism 1996; Suppl.: 69-75 
${ }^{81}$ Litten RZ, Allen J, Fertig J. Pharmacotherapies for alcohol problems: a review of research with focus on developments since 1991. Alc Clin Exp Res 1996; 2: 859-876

82 Gorelick DA, Paredes A. Effect of fluoxetine on alcohol consumption in male alcoholics. Alc Clin Exp Res 1992; 16: 261-265

83 Janiri L, Gobbi G, Mannelli P, Pozzi G, Serretti A, Tempesta E. Effects of fluoxetine at antidepressant doses on short-term outcome of detoxified alcoholics. Int Clin Psychopharmacol 1996; 11: 109-117

${ }^{84}$ Malcolm R, Anton RF, Randall CL, Johnston A, Brady K, Thevos A. A placebo-controlled trial of buspirone in anxious inpatient alcoholics. Alc Clin Exp Res 1992; 16: 1007-1013

${ }^{85}$ Tollefson GD. Anxiety and alcoholism: a serotonin link. British Journal of Psychiatry, 1991: Suppl.: 34-39

\section{Dr. Jens Wittfoot}

Psychiatrische Klinik

Krankenanstalten Gilead, Bethel

Remterweg 69-71

33617 Bielefeld

E-mail: wittfoot@psychiatrie.gilead.de 